\title{
Energy Aware Heuristic Approach for Cluster Head Selection in Wireless Sensor Networks
}

\author{
A. Tamizharasi M. E. ${ }^{\star 1}$, J. Jasmine Selvathai M. E. ${ }^{2}$, A. Kavi Priya ${ }^{3}$, Maarlin R. ${ }^{4}$, \\ Harinetha $M .^{5}$ \\ ${ }^{1,2}$ Department of Computer Science and Engineering, RMD Engineering College, Chennai, India \\ ${ }^{3,4,5}$ Department of Computer Science and Engineering, Knowledge Institute of Technology, Salem, India \\ *Corresponding author, email: tamizh4384@gmail.com', jjasmineselvathai@yahoo.co.in², \\ kavipriya3597@gmail.com ${ }^{3}$, maarlinrozario@gmail.com ${ }^{4}$, harinetha123@gmail.com ${ }^{5}$
}

\begin{abstract}
Wireless sensor networks idea is envisioned and defined as self-deployed, error prone, long living inexpensive communication devices that are densely deployed to collect data from physical space. Sensor nodes communicate with each other to detect events depending on the application, to collect and process data, and to transmit the sensed information to the base station by hopping the data from node to node. Major challenge in wireless network is energy consumption, since the sensor devices are deployed on unattended wide areas and replacing their batteries is not very feasible. Therefore, designing energyaware algorithms becomes a major challenge for extending the lifetime of sensors. The lifetime of the network can be maximized by selecting the most optimal cluster head. In this paper, a heuristic decision making approach is proposed for producing energy-aware clusters with optimal selection of cluster head. This helps to improve the efficiency and accuracy and overcome the drawbacks like getting trapped at a local extreme in the optimization process.
\end{abstract}

Keywords: wireless sensor networks, energy-aware clusters, LEACH, PSO, cluster head

\section{Introduction}

Wireless sensor networks consist of collection of wireless devices that can communicate without a wired infrastructure. Each device can sense, talk and communicate with its peers. Sensor nodes are energy constrained therefore energy aware algorithms is an important factor for preserving their life time. Clustering is a standard procedure in multivariate data analysis used for energy efficient communication and is designed to explore an inherent natural structure of the data objects. Clustering methods has a wide range of applications, such as medical research, psychology, economics and pattern recognition.

Clusters are formed by grouping of sensor nodes. Every cluster has a leader, referred as Cluster Head $(\mathrm{CH})$ which is selected or pre-assigned by the network designer. A CH can schedule activities in the cluster and can aggregate the data collected by the sensors in its cluster.Sensors are randomly deployed in such a way that it is within the transmission range, which enables certain nodes to enter the sleep mode which in turn saves significant energy. Each node transmit its information to the head of cluster, and it collects the data from all other nodes of the cluster, then it compress and format the data before sending it to the base station (BS). The optimal cluster head is the one which has the highest residual energy, the maximum number of neighbor nodes, and nearest from the base station. So the cluster based approach has the advantage of enhanced resource allocation and bandwidth reusability.

The $\mathrm{CH}$ consumes more power than the other nodes, because of the load on it. The connectivity path between the $\mathrm{CH}$ and base station is ensured by the position of the $\mathrm{CH}$ which makes it crucial in terms of energy efficiency and performance. This approach saves energy of transmission to the base station and also the time of transmission, selection and retransmission of the sensed data.The lifespan of the sensor network and the coverage of clustering techniques depend on the node deployment, the network architecture, the characteristics of $\mathrm{CH}$ nodes and the network operation model.

Buddha Singh et al proposed a PSO approach for optimizing the location of the cluster head at reduced cost [1]. A semi distributed approach is used and implemented the algorithm 
within the cluster rather than the base station. The residual energy, distance and head count is used as the selection criteria for the objective function. A fuzzy logic for the selection of cluster head has been applied in [2]. The Fuzzy multiple attribute decision-making (MADM) approach is used for choosing the cluster heads which helps to improve the network lifetime in homogeneous environments.

Low-energy adaptive clustering hierarchy protocol architecture for microsensor networks is presented in [3]. The proposed architecture combines the ideas of energy-efficient cluster-based routing and media access with application-specific data aggregation for achieving system lifetime and quality. LEACH enables self-organization of large numbers of nodes, efficient methods for managing the clusters and rotating cluster head positions to distribute the energy load evenly among the nodes.Random Competition based Clustering algorithm (RCC) applies the First Declaration Wins rule, in which any node can monitor the rest of the nodes in its transmission range if is the first to claim to be a cluster head $(\mathrm{CH})$. Concurrent broadcast of $\mathrm{CH}$ claims may create conflict. RCC explicitly employs a random timer and uses the node ID for arbitration. Hierarchical control clustering has two phases: Breadth First Search tree discovery and cluster formation. Multiple nodes can initiate the cluster formation [3]. Energy Efficient Hierarchical Clustering (EEHC) is a distributed clustering algorithm where each sensor node announces itself as a $\mathrm{CH}$ with probability $\mathrm{p}$ to the neighboring nodes in its communication range.

Development of a centralized, energy aware cluster-based protocol extends the sensor network lifetime by using PSO algorithm in such a way that it makes use of a high-energy node as a cluster head and produces clusters that are evenly positioned throughout the whole sensor field [4]. PSO has the characteristics of simplicity, fast convergence and memorizing in both position and velocity information in the procession of evolution to propose a mobile beacon node path access method [5]. Vipin pal et.al proposed genetic algorithm to select the cluster head centralized clustering algorithms for achieving a load balanced network [7]. The cluster head is selected according to their residual energy and takes care of trade-off of inter- and intracluster communication distance. Genetic algorithm helps to optimize the number of cluster head. Section II describes the proposed work. Section III discusses simulation environment and Section IV describes conclusion.

\section{Proposed Work}

Clustering is designed to explore an inherent natural structure of data objects and is mostly used in multivariate data analysis. Cluster formation and cluster head selection techniques are employed to achieve better operation and the prolong network lifetime by minimizing energy consumption. LEACH (Low Energy Adaptive Clustering Hierarchy) includes a new distributed cluster formation technique that enables self-organization of large number of nodes, algorithms for adapting clusters and rotating cluster head positions to evenly distribute the energy load among all the nodes.

In PSO (Particle Swarm Optimization) approach [5], the selection criteria of the objective function are based on the residual energy, minimum average distance from the member nodes and head count of the probable head nodes. PSO approach attempts to select the cluster head which can minimize the intra cluster distance between itself and the cluster member, and the optimization of energy management of the network. But one of the limitations with the PSO algorithm is that it is possible to get trapped at local optimum solution. To overcome this in the proposed approach the Bacterial Foraging Algorithm (BFA) and PSO is hybridized to improve the performance.

\subsection{LEACH}

LEACH protocol includes distributed cluster formation to increase the life span of the network. LEACH randomly selects One node in the cluster as the cluster head $(\mathrm{CH})$ and the others are members of the cluster.

The $\mathrm{CH}$ collects the information from the other nodes, aggregate the data and then transmit to the base station. LEACH randomly rotates the $\mathrm{CH}$ to evenly distribute the energy load among the sensors to save the battery of the individual node thereby increasing the lifetime of the network. LEACH uses a TDMA/CDMA MAC to reduce inter-cluster and intra-cluster 
collisions. After specified time interval, randomized rotation of cluster-head is conducted so that the energy dissipates uniformly in the sensor network.

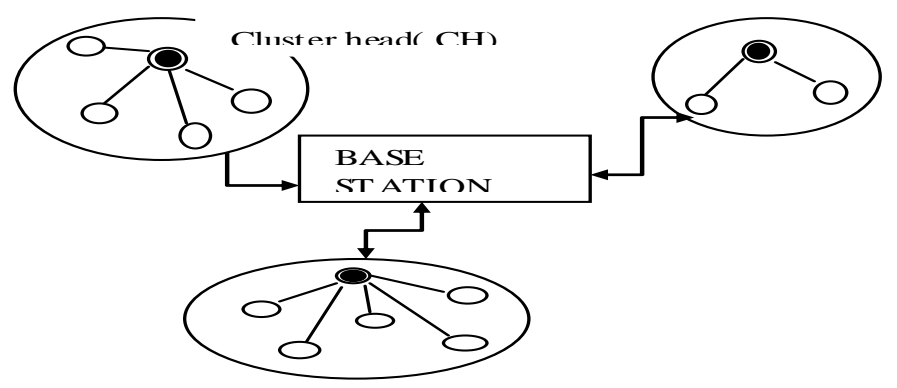

Figure 1. LEACHI for Wireless Sensor Networks

The operation of LEACH consists of two phases, the set-up phase and the steady state phase. In the set-up phase, the clusters are framed and cluster-heads are selected based on the probability function. Every node selects a random number between 0 and 1 and if it is less the threshold value $T$ then it becomes the cluster head for the current round. The threshold value is calculated based on the equation

$$
\mathrm{T}(\mathrm{n})=\mathrm{P} / 1-\mathrm{P}\left(\mathrm{r} \bmod \left(\frac{1}{\mathrm{P}}\right)\right), \text { if } \mathrm{n} \text { in } \mathrm{G}
$$

which includes the desired percentage $(P)$ to be a cluster-head, the current round $(r)$, and the set of nodes that have not been selected as a cluster-head in the last (1/P) rounds, denoted G.

In the steady state phase, the aggregated data is transmitted to the BS. The cluster communication is through different CDMA codes in order to reduce the interference from nodes belonging to other clusters. After a certain time, the network goes back into the set-up phase again and enters another round of selecting new cluster-heads.

\subsection{PSO}

In PSO application a centralized head is used to maintain the attributes of the member nodes for every generation. In order to accomplish the requirement, a randomly selected Cluster Assistant ( $\mathrm{nCA}$ ) sensor node is assigned in every cluster to maintain the position of every sensor along with other sensor characteristics, like - position, energy, number of cluster head, global best and additional PSO parameter values acts as the local processing centre to a cluster. At the beginning of every round of cluster head selection, all the sensor nodes in the cluster provides their initial location, energy level and number of nodes present to the assistant node [1].

PSO models the social behavior of a flock of birds [6] which is used applied for solving the optimization problem. In a $n$ - dimensional search space, $s$ number of sensor nodes are randomly deployed. Each node $\mathrm{i}$ occupies the position xid and vid where $1 \leq \mathrm{i} \leq \mathrm{s}$ and $1 \leq \mathrm{d} \leq \mathrm{n}$. Each node is evaluated through an objective function $f(x 1, x 2 \ldots x n)$, where $f: R n \rightarrow R$. The cost or fitness of the sensor node close to the global solution is lower (higher) than that of the node that is away. PSO thrives to minimize (maximize) the fitness function. In the global-best version of PSO, the position where the node $i$ has its lowest cost is stored as (pbestid) and gbestd, the position of the best node in the entire search space. In each iteration k, velocity $\mathrm{Vi}$ and position Xi are updated using (1) and (2) and continued until either an acceptable gbest is achieved or a fixed number of iterations kmax is reached.

$$
\begin{aligned}
& \mathrm{Vi}=\mathrm{Vi}-1+\mathrm{c} 1 * \operatorname{rand}() *(\text { pbest }-\mathrm{Xi})+\mathrm{c} 2 * \operatorname{rand}() *(\text { gbest }-\mathrm{Xi}) \\
& \mathrm{Xi}=\mathrm{Xi}-1+\mathrm{Vi}
\end{aligned}
$$

$\mathrm{Vi}$ is the node's velocity. Initially the values of the velocity are randomly generated within the range $[-\mathrm{Vm}, \mathrm{Vm}]$ where $\mathrm{Vm}$ is the maximum value that can be assigned to any $\mathrm{Vi}$. $\mathrm{Xi}$ 
is the nodes current position. pbest and gbest are the particle and swarm best values. rand () is a random number between 0 and 1. $\mathrm{c} 1, \mathrm{c} 2$ are learning factors governing the cognition and social components. Usually $\mathrm{c} 1=\mathrm{c} 2=2$.

\subsection{BFA-PSO}

The main objective of the proposed hybrid approach is to determine the optimized position of the randomly deployed nodes in the search space that maximizes the fitness function $f(\lambda)=\log P(o / \lambda)[6]$. The fitness function is used to determine which solution is better than the others and is instrumental in determining the direction as well as magnitude of the velocity vector in each iteration. From the E. Coli bacteria's mechanism of finding the places with higher nutrient value and avoiding noxious places, an optimization technique that models this process, namely Bacterial Foraging Algorithm is evolved. BFA is a population-based numerical optimization algorithm. It has been applied for solving practical engineering problems like optimal control, harmonic estimation, channel equalization etc [4].

Chemotaxis is a foraging behavior that captures the process of optimization, where the nonanchor (target) nodes try to climb up to the transmission range. From its current position, target node will move to the position that has a minimum fitness value. The "speed" of the node movement is controlled by the chemotactic step size, C. Change in the direction of movement is tumbling whereas moving in the same direction is swimming. The direction of movement after tumble is based on the position of every node and its velocity. At each iteration i, nodes velocity vi and position xi of each node are updated using (1) and (2) in PSO.

Thus, the movement of target node from one position to another position will be the sum of current position and the step size and direction generated from PSO. By this the nodes will move towards the global optimum position every time. If at target node position, the fitness value is lower than the previous value then the target node will move one step in the same direction with the step size and is continued until a minimum fitness value is reached but only for a certain number of steps, Ns. After swims, the nodes have to tumble. Target nodes will reproduce very fast if the RSSI is high and will die if less so that the population size will decrease significantly. This mechanism keeps the node's population constant.

To model the reproduction mechanism, after Nch chemotactic step size, the fitness values of all the target nodes are sorted in ascending order based on their accumulated cost function value. After $\mathrm{Nr}$ reproduction steps, the target nodes which have probability value (between 0 and 1) lower than certain threshold value (ped) are eliminated and dispersed to another location and nodes which have probability value higher than ped keep their current position. After elimination and dispersal event, node will start chemotaxis until maximum reproduction steps are achieved and then followed by other elimination and dispersal events. This routine is done until maximum Ne elimination and dispersal events are achieved

Algorithm 1 Hybrid BFA-PSO

1. Initialize $\mathrm{Nch}, \mathrm{Nr}, \mathrm{Ne}, \mathrm{Ns}, \mathrm{C}, \mathrm{V}, \mathrm{X}$

2. Elimination-Dispersal loop: while $\mathrm{I}<=\mathrm{Ne}$ do

3. $|=|+1$

4. Reproduction loop : while $\mathrm{m}<=\mathrm{Nr}$ do

5. $\quad \mathrm{m}=\mathrm{m}+1$

6. Chemotaxis loop : If $\mathrm{n}<=\mathrm{Nch}$

7. For each node $\mathrm{i}$

8. While $(\mathrm{sm}<\mathrm{Ns})$ do

9. $\quad \mathrm{sm}=\mathrm{sm}+1$

10. Compute $\mathrm{Vi}=\mathrm{Vi}-1+\mathrm{c} 1 * \operatorname{rand}() *($ pbest $-\mathrm{Xi})+\mathrm{c} 2 * \operatorname{rand}() *$ (gbest $-\mathrm{Xi}$ )

11. Compute $\mathrm{Xi}=\mathrm{Xi}-1+\mathrm{Vi}$

$12 . \quad$ If $(f(X i)>f(X i-1))$

$13 . \quad$ Let $f(X i)=f(X i-1)$

$14 . \quad$ Compute new $X i$

$15 . \quad$ Else

16.

$17 . \quad$ End if

18. End while 
19. Compute jfit

20. Eliminate node having highest jit and split the other nodes into two at the same position

21. End for

22. End if

23. End while

24. Eliminate the node having ped and create new node at a random position

25. End while

\section{Simulation \& Results}

Simulation of the WSN and its performance evaluation have been implemented in NS2. The sensor nodes are deployed randomly in a sensor field having dimensions of 1000 $\times 1000$ sqm. The transmission range of the anchor node is $200 \mathrm{~m}$. The General Network Topology Parameters are:

\begin{tabular}{cl} 
Table 1. General Network & Topology Parameter \\
\hline Channel Type & Wireless Channel \\
\hline Radio-Propagation Model & TwoRayGround \\
Antenna Model & Omni Antenna \\
Packet Size & 512 bits \\
Network Layer Protocol & LEACH/PSO/BFA-PSO \\
Total no. of nodes & 100 \\
Total Simulation Time & 200 \\
Model & Energy Model \\
\hline
\end{tabular}

The probability is set to 0.90 in LEACH implementation. In PSO and hybrid approach implementation, Cluster radius is calculated as Network Radius / sqrt (no of clusters ) and the total number of iterations is 100 . The following Performance Metrics are analyzed:

- Average Residual Energy

- No of Nodes Alive

- No of Cluster Head Elected

- Average Energy Consumption

Figure 2 shows the variation in Average Residual Energy with respect to the number of nodes deployed. For every 25 nodes increase in the deployment, the average residual energy of the nodes in LEACH approach gets decreased by $19 \%$ and in PSO by $16.7 \%$ and hybrid algorithm by $13 \%$. It shows that hybrid (BFA-PSO) approach creates energy efficient clusters than LEACH implementation.

No. of Nodes Alive by varying Simulation Time is shown in Figure 3. For the increase in simulation time, total number of nodes alive in hybrid (BFA-PSO) implementation is higher than of LEACH implementation which results in long span of network lifetime. Figure 4 depicts the No. of cluster heads elected with respect to the simulation time. The iteration based BFA-PSO approach produces more stable number of cluster heads with long alive nodes than the probability based LEACH approach.

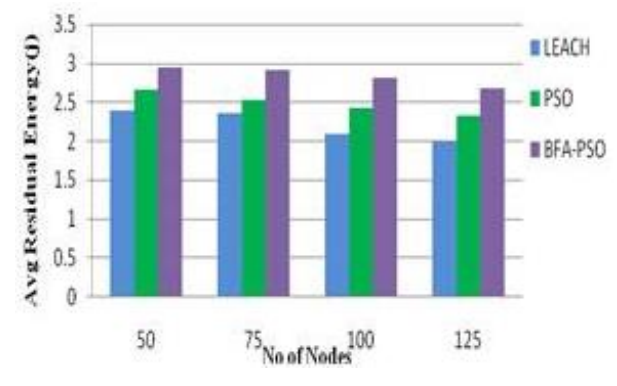

Figure 2. No. of nodes vs average residual energy

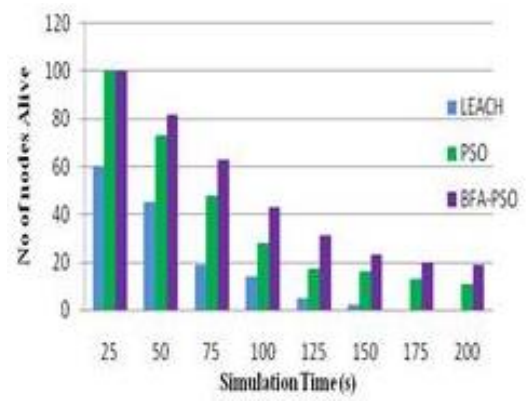

Figure 3. No. of nodes alive vs simulation time 
Energy Consumption. For the every increase of 25 nodes deployment LEACH implemented energy aware clusters produce 19\% of increase in energy consumption as compared with our proposed BFA-PSO implemented clusters which produce $5 \%$ of increase in energy consumption. BFA-PSO optimizes the intra-cluster distance and reduces energy consumption of the network. Figure 5 shows no. of nodes vs average energy consumption

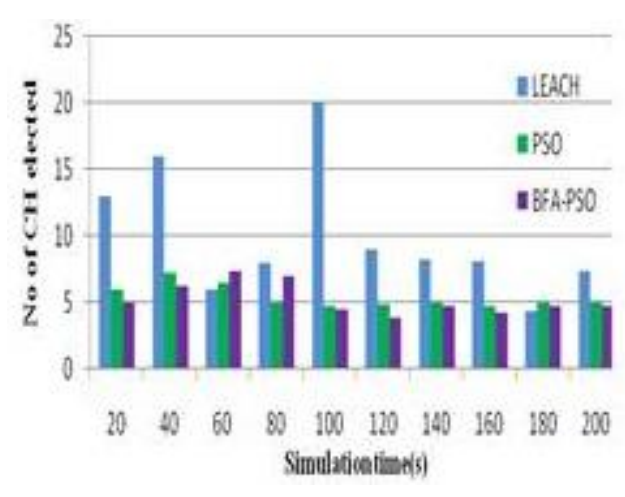

Figure 4. No. of $\mathrm{CH}$ s elected vs simulation time

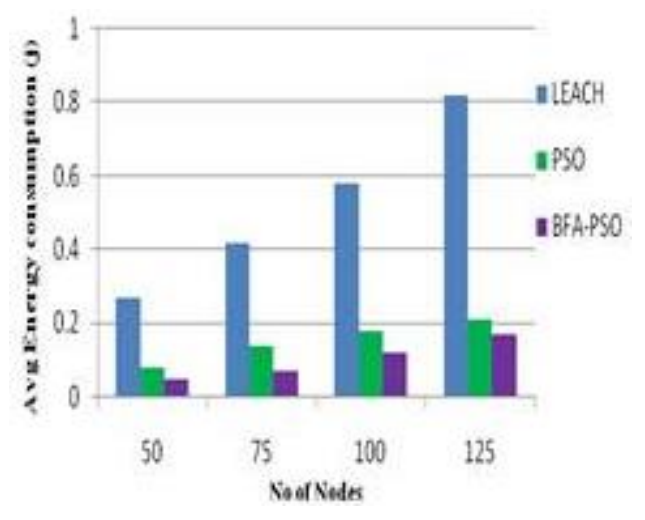

Figure 5. No. of nodes vs average energy consumption

\section{Conclusion}

Energy aware cluster head selection using LEACH, PSO and hybrid BFA-PSO are implemented in the wireless sensor networks. The selection of a cluster head using the hybrid approach minimizes the intra cluster distance between cluster head and the cluster member, and the optimization of energy management of the network. The premature convergence of PSO approach is overcome by using Bacterial Foraging Algorithm. From the simulation results, it is seen that Energy-aware Optimal cluster head selection using BFA-PSO approach increases the network lifetime of the cluster in such a way by reducing the total energy consumption than LEACH implementation.

\section{References}

[1] Buddha Singh, DK Lobiyal. Energy-aware cluster head selection using particle swarm optimization and analysis of packet retransmissions in WSN. C3IT-2012 IEEE/ACM Transactions on Computational Biology and Bioinformatics. 2011; 8: 171-176

[2] Puneet Azad, Vidusi Sharma. Cluster Head Selection in Wireless Sensor Networks under Fuzzy Environment. ISRN Sensor Networks, Hindawi Publishing Corporation. 2013.

[3] Heinzelman W, Chandrakasan A, Balakrishnan H. An application-specific protocol architecture for wireless microsensor networks. Wireless Communications, IEEE Transactions. 2002; 1(4): 660-670.

[4] Ameer Ahmed Abbasi, Mohamed Younis. A survey on clustering algorithms for wireless sensor networks. Science Direct Computer Communications. 2007; 30: 2826-2841

[5] Latiff NMA, Tseminidis CC, Sheriff BS. Energy aware clustering for wireless sensor networks using particle swarm optimization. The 18th annual IEEE International Symposium on Personal, Indoor and Mobile Radio Communications (PIMRC'07). 2007: pp1-5

[6] Hu B, Liu S, Li H. Mobile Beacon Node Path scheme Based on Particle Swarm Optimization in Wireless Sensor Networks. ICEMI'09. 2009;3-306-3-309

[7] Vipin Pala, Yogita, Girdhari Singh, RP Yadav. Cluster Head Selection Optimization Based on Genetic Algorithm to Prolong Lifetime of Wireless Sensor Networks. Third International Conference on Recent Trends in Computing (ICRTC 2015), Procedia Computer Science 57. 2015: 1417-1423.

[8] The Network Simulator -ns2, URL: http://www.isi.edu/nsnam/ns/ 